\title{
.
}

\section{Promises and Challenges: Recent Development of Granular FePt-L10 Thin Film Material for Heat Assisted Magnetic Recording}

\section{ISSN: 2576-8840}

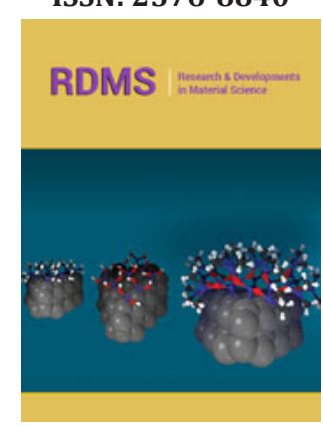

*Corresponding author: Jian-Gang Zhu, ABB Professor of Engineering and IEEE Fellow, Data Storage Systems Center, Department of Electrical and Computer Engineering, Carnegie Mellon University, USA

Submission: 些April 18, 2019

Published: 阱 April 22, 2019

Volume 10 - Issue 4

How to cite this article: Jian-Gang Zhu. Promises and Challenges: Recent Development of Granular FePt-L10 Thin Film Material for Heat Assisted Magnetic Recording. Res Dev Material Sci. 10(4). RDMS.000745.2019.

DOI: $10.31031 /$ RDMS.2019.10.000745

Copyright@ Jian-Gang Zhu, This article is distributed under the terms of the Creative Commons Attribution 4.0 International License, which permits unrestricted use and redistribution provided that the original author and source are credited.

\section{Jian-Gang Zhu*}

Department of Electrical and Computer Engineering, Carnegie Mellon University, USA

\section{Opinion}

At room temperature, perpendicularly ordered $\mathrm{L} 1_{0} \mathrm{FePt}$ thin film exhibits extremely high uniaxial anisotropy with easy axis oriented along the ordering direction [1]. The relatively low curie temperature and high temperature gradient of the anisotropy field near the Curie point of the L1 $1_{0}$ FePt makes it almost perfect as a recording media for heat assisted magnetic recording (HAMR) technology [2-5]. In HAMR, an optically (by laser illumination) excited plasmonic near field transducer provides localized electric field to the recording media as schematically shown in Figure 1. The localized alternating electric field at optical frequency generates rapid electronic motion in the metallic magnetic grains of the recording medium underneath, heating up the grains over their Curie temperature. As the medium moves away from the NFT, thereby, the localized electric field, the temperature start to cool down, the medium grains start to regain its magnetization with orientation guided by the recording head field. As the temperature of the medium grains decreases, the magnetic anisotropy of the grains quickly rises. When it exceeds the capability of the recording field, the magnetization direction freezes [6]. In order to insure sharp magnetic transitions between recorded bits, the lateral size of medium magnetic grains needs to be small with thermally insulating grain boundaries. A relatively thick heat sink layer is placed underneath the magnetic layer to facilitate a high thermal gradient, currently at $10 \mathrm{~K} / \mathrm{nm}$, which is essential to suppress the transition jitter caused by grain-to-grain Curie temperature variation in the media [7-10].

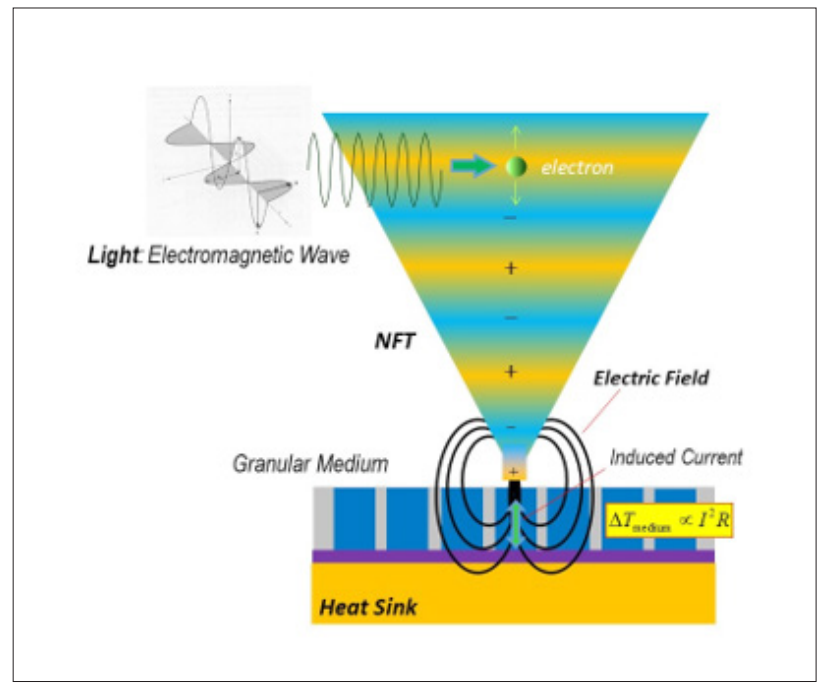

Figure 1: Illustration of Heat-Assisted Magnetic Recording with an optically excited plasmonic near-field transducer and FePt-L10 granular thin film media.

In order to ensure all the FePt grains are ordered in the direction perpendicular to film plane, well textured polycrystalline $\mathrm{MgO}$ layer with (002) orientation has been commonly used as the immediate underlayer for the FePt layer. Since the MgO lattice constant is slightly greater than that of the FePt, $\sim 9 \%$, it provides a tensile stress to the FePt lattice to facilitate 
perpendicular ordering since the c/a ratio of a FePt unit cell becomes smaller than unity when it is ordered in $\mathrm{L} 1_{0}$ phase [11]. This underlayer technique is particularly effective when FePt grain size is relatively small, currently around $7 \mathrm{~nm}$, while $\mathrm{MgO}$ grain size is relatively large. However, during film deposition process, if a FePt grain grows laterally across a MgO grain boundary, the extension of the lattice coherence in the FePt grain could mean losing the lattice coherence with the neighboring MgO grain underneath. The loss of the tensile stress may cause the portion of the FePt grain on the different MgO grain to order along one of the in-plane directions instead, yielding the grain multi-variant. This multi-variant formation mechanism has been experimentally identified in a systematic nano-diffraction TEM study [12]. To mitigate the effect, using a thick (002) textured $\mathrm{Cr}$ or $\mathrm{CrRu}$ underlayer beneath the $\mathrm{MgO}$ layer to significantly increase $\mathrm{MgO}$ grain size has been utilized to reduce the occurrence of such multi-variant grains.

In order to achieve high degree of ordering, FePt-L1 $1_{0}$ granular layer needs to be deposit at elevated substrate temperature during sputtering process, usually above $500{ }^{\circ} \mathrm{C}$. At such relatively high temperature, it has been very challenge to make the magnetic layer with desired film microstructure:

a) Well-defined encircled non-metallic grain boundaries;

b) Uniformly distribution of FePt grain size;

c) Column FePt grain with relatively high aspect ratio (currently grain height to grain diameter ratio is $h / D \sim 2$ ).

Over the past decade, the development of the FePt-L1 $1_{0}$ based HAMR media have been focused on achieving the above three objectives in optimizing microstructure [13]. The fully encircled non-metallic grain boundary is important for thermal isolation to prevent lateral heat flow in maintaining high thermal gradient. Many grain boundary materials have been studied experimentally. Various oxides, such as SiOx, as grain boundary do not produce fully encircled grain boundaries surrounding FePt grains, but rather yield maze-like or worm-like FePt grains. On the other hand, carbon as the grain boundary material has had good success in terms of yielding microstructures with completely encircled FePt grains [14]. FePt grain size can be controlled by the volumetric ratio between carbon and FePt during deposition. However, with carbon as grain boundary material, the nucleation density of FePt grains appears to be limited, preventing reduction of FePt grain pitch to go below $6 \mathrm{~nm}$. Furthermore, carbon grain boundaries do not seem to stop continued FePt grain nucleation on the $\mathrm{MgO}$ underlayer during the entire magnetic layer deposition process, resulting relative broad grain size distribution or even bi-modal distribution. The penetration of FePt atoms through carbon grain boundaries during the entire deposition process also yields extensive lateral growth all the way through the depth. The lateral grain growth significantly limits the grain height. More recently, with using alternative grain boundary material, higher height to diameter aspect ratio has been achieved [15]. The ability to produce taller grains with continued reduction of grain size is crucial for suppressing transition jitter caused by thermal agitation during the recording process when temperature is substantially higher than ambient. At small grain sizes, Curie temperature becomes grain size dependent due to significant surface-to-volume ratio with smaller grain diameter having lower Curie temperature. Therefore, a grain size distribution will directly translate into grain-to-grain Curie temperature variation. Such variation can cause significant transition jitter, becoming a limiting factor for linear recording density. Theoretical modelling study shows that the relative standard deviation of Curie temperature distribution needs to be kept below 2\% for HAMR to surpass the area density capability of conventional perpendicular magnetic recording (CPMR) [16].

Since the grain size dependence of Curie temperature arises from the fact that atomic spins at grain boundary surface are missing more than half of its neighbours comparing to the spins in the interior of the grains. A thermally insulating magnetic grain boundaries with Curie temperature higher than bulk L1 $1_{0}$ FePt could alleviate the problem provided such grain boundary material will also facilitate the required film microstructure. For this purpose, mixing $\mathrm{BaFeO}$ material with carbon as a novel grain boundary material has been investigated by co-sputtering them with FePt [17]. The study found that granular FePt-L1 $1_{0}$ with the mixed grain boundary material exhibits higher Curie temperature than that of pure carbon grain boundaries with the same average FePt grain size as well as reduction of Tc distribution, although small.

Although challenging, the development of FePt-L1 $1_{0}$ granular thin film as HAMR media has made substantial advancement towards the expected area density capability performance [15].

\section{References}

1. Weller D, Moser A, Folks L, Best ME, Lee W, et al. (2000) High ku materials approach to 100 gbits/in2. IEEE Trans Magn 36(1): 10-15.

2. Kryder MH, Edward CG, Terry WM, William AC, Robert ER, et al. (2008) Heat assisted magnetic recording. Proc IEEE 96(11): 1810-1835.

3. Challener WA, Chubing P, Itagi AV, Karns, Wei Peng, et al. (2009) Heatassisted magnetic recording by a near-field transducer with efficient optical energy transfer. Nature Photon 3: 220-224.

4. Stipe BC (2010) Magnetic recording at $1.5 \mathrm{Pbm}^{2}$ using an integrated plasmonic antenna. Nature Photon 4: 484-488.

5. Seigler MA, William AC, Edward G, Nils G, Ganping J, et al. (2007) Integrated heat assisted magnetic recording head: design and recording demonstration. IEEE Trans Magn 44(1): 119-124.

6. Zhu JG, Li H (2013) Understanding signal and noise in heat assisted magnetic recording. IEEE Trans Magn 49(2): 765-772.

7. Ju G, Yingguo P, Eric KCC, Alexander QW, Xiaobin Z, et al. (2015) High-density heat-assisted magnetic recording media and advanced characterization-progress and challenges. IEEE Trans Magn 51(11): 3201709.

8. Acharya R (2014) Challenges and recent development of heat assisted magnetic recording media. In Proc TMRC, pp. 57-58.

9. Zhu JG, Li H (2015) SNR impact of noise by different origins in FePt-L1 HAMR media. IEEE Trans Magn 51(4): 15142770.

10. Pisana S, Jain S, Reiner JW, Parker GJ, Poon CC, et al. (2014) Measurement of the Curie temperature distribution in FePt granular magnetic media. Appl Phys Lett 104(16): 162407.

11. Peng Y, Zhu JG, Laughlin DE (2006) L1 FePt-MgO perpendicular thin film deposited by alternating sputtering at elevated temperature. J Appl Phys 99(8): 08F907. 
12. Ho H, Zhu J, Kulovits A, Laughlin DE, Zhu JG (2014) Quantitative transmission electron microscopy analysis of multi-variant grains in present L1 -FePt based heat assisted magnetic recording media. J Appl Phys 116(19): 193510.

13. Qin Y, Zhu JG (2019) Impact of magnetic medium grain height in heatassisted magnetic recording. IEEE Magnetics Letts 10: 6502305.

14. Hono K (2018) Heat-assisted magnetic recording media materials. MRS Bull 43(2): 93-99.
15. Kubota Y (2018) Heat-assisted magnetic recording's extensibility to high linear and area density. IEEE Transactions on Magnetics 54(11): 320126.

16. Weller D, Gregory P, Oleksandr M, Eric C, Barry S, et al. (2014) A HAMR media technology roadmap to an areal density of $4 \mathrm{~Tb} / \mathrm{in}^{2}$. IEEE Trans Magn 50(1): 3100108.

17. Zhou B, Varaprasad, Dai Z, Laughlin DE, Zhu JG (2018) The effect of adding a magnetic oxide in the grain boundaries of HAMR media. Appl Phys Lett 113(8): 082401.

For possible submissions Click below: 